Revista de Investigación Educativa 26

enero-junio, 2018 | ISSN 1870-5308 | Xalapa, Veracruz

Instituto de Investigaciones en Educación | Universidad Veracruzana

\title{
Relaciones sociales de discriminación en espacios de aprendizaje en la carrera de Psicología a distancia en la Universidad Nacional Autónoma de México
}

\section{Social Relations of Discrimination in Learning Spaces in Universidad Nacional Autónoma de México Distance Psychology Faculty}

\section{Marco Antonio González Pérez}

Recibido: 21 de octubre de 2016 Aceptado: 13 de diciembre de 2017

Para identificar relaciones discriminatorias en la carrera de Psicología a distancia de la Universidad Nacional Autónoma de México, se aplicó cuestionario de 20 reactivos a 673 estudiantes (72.5\% mujeres y 27.5\% hombres). Se indagó la discriminación en espacios de aprendizaje, roles, testigos y sus razones. Los resultados indicaron que no existe discriminación generalizada en espacios de interacción, pero se observó que la plataforma Moodle es donde más sucede. $22.7 \%$ señaló haber sido testigo de discriminación alumno-alumno y 17.1\% profesor-alumno y 15.3\% sufrió discriminación por su forma de pensar. Se refirieron casos de discriminación profesor-alumno, alumno-alumno y de instituciones externas a alumnos. En este estudio, los fundamentos de la discriminación se basan, principalmente, en la psicología social, la sociología y antropología. Los resultados, aunque obtenidos en una universidad concreta, muestran la necesidad de que instituciones que imparten carreras a distancia, consideren la posible ocurrencia de discriminación en sus aulas de aprendizaje.

Palabras clave: Educación a distancia; Tecnologías de la Información y la Comunicación; discriminación social.

\footnotetext{
a Doctor en Psicología, Profesor Titular "A" de Tiempo Completo, Facultad de Estudios Superiores de Iztacala, Universidad Nacional Autónoma de México, México. 
To identify discriminatory relationships in Psychology on line career at the National Autonomous University of Mexico, a questionnaire of 20 items was applied to 673 students ( $72.5 \%$ women and $27.5 \%$ men). Discrimination was investigated in learning spaces, roles, witnesses and their reasons. The results indicated that there is no generalized discrimination in interaction spaces, but it was observed that the Moodle platform is where most happens. $22.7 \%$ said that they had witnessed student-student discrimination and $17.1 \%$ teacher-student and $15.3 \%$ suffered discrimination because of their way of thinking. Cases of teacher-student, student-student and external institutions to students were mentioned. In this study theoretical fundament of discrimination are based, mainly, on social psychology, sociology and anthropology. Results, although obtained in a specific university, show the need for institutions, that teach on line careers, to consider possible occurrence of discrimination in their learning rooms.

Keywords: Distance education; Communication and Information Technologies; social discrimination.

\title{
Relaciones sociales de discriminación en espacios de aprendizaje en la carrera de Psicología a distancia en la Universidad Nacional Autónoma de México ${ }^{1}$
}

\author{
Social Relations of Discrimination in Learning Spaces in Universidad \\ Nacional Autónoma de México Distance Psychology Faculty
}

\section{Introducción}

【a realización de diagnósticos para conocer el estado que guarda un modelo o sisoportunidad que sustenten intervenciones de mejora. En el Sistema de Universidad

1. Investigación realizada gracias al Programa UNAM-DGAPA-PAPIIT IN 307616. 
Abierta y Educación a Distancia (suayed) Psicología de la Facultad de Estudios Superiores Iztacala de la Universidad Nacional Autónoma de México (UNAM), se han realizado algunos ejercicios participativos de este tipo. Los temas evaluados de acuerdo con González (2015a) fueron: trámites administrativos (con los subtemas: servicio social, prácticas, credencialización, titulación, áreas de profundización y trámites diversos); ambiente académico (con los subtemas: relación tutor-alumno, formas de evaluación); permanencia y egreso(con los subtemas: plan de estudios y programas, carga académica y deserción escolar); carrera académica (con los subtemas: plan de carrera, actualización académica, investigación, profesionalización de la plantilla docente y vida colegiada) y aula virtual y TIC en el alumno SUAyED (con los subtemas: plataforma tecnológica y aplicaciones educativas de la web, técnicas pedagógicas y didácticas en el aula virtual).

Los encuentros diagnósticos de alumnado y profesorado denominados "Fortaleciendo el suAyed Iztacala" se llevaron a cabo en marzo y abril de 2014 y arrojaron información relevante que mostró que, entre otros, existían problemas muy sensibles en la interacción profesor-alumno. Tal como se señala en la siguiente cita:

En la discusión de la mesa II Ambiente académico, los participantes encontraron un gran número de problemáticas que afectan el devenir de su carrera profesional como los siguientes: falta de espacios para ampliar la formación académica de los alumnos, inflexibilidad del tutor para recibir tareas entregadas fuera de tiempo, exámenes con reactivos mal diseñados e inválidos y rechazo de la Coordinación para revisar exámenes finales, abandono tutorial de las clases y escasa comunicación, inexactitudes en los calendarios del curso entregados por el profesor, falta de identidad del alumno suAyed con la universidad, excesiva carga de trabajo de los alumnos, rechazo de los profesores a aceptar correcciones en trabajos y tareas, instrucciones confusas en las actividades a realizar y carencia de un espacio para reportar incidencias en la relación tutor-profesor. (González, 2015a, p. 64)

Se comprobó entonces que algunos alumnos se sentían maltratados, poco valorados o abiertamente discriminados por el profesor y la administración. Este ejercicio diagnóstico fue el antecedente del presente estudio. 


\section{Fundamentación}

La discriminación, como es bien sabido, es un fenómeno social ampliamente difundido en México y que tiene siglos de existencia. Es un producto sociocultural e histórico que, lamentablemente, regula las relaciones sociales entre individuos y grupos y que determina la falta de cohesión y fomenta la desigualdad en nuestro país.

Una aproximación, desde la disciplina psicológica, a la discriminación social, la presenta, desde la psicología social, el desaparecido profesor Tajfel (1984) por medio de sus estudios de diferenciación intergrupal llevados a cabo en la década de los setenta del siglo pasado. A partir de ellos establece que la diferenciación grupal alcanzada por el favoritismo endogrupal y la discriminación exogrupal, por medio de procesos de comparación social, es un mecanismo ineludible que utilizan los miembros de los grupos para dotarse de una identidad social positiva.

Sin embargo, el establecimiento de diferencias no se traduce, necesariamente, en exacerbación de las mismas. La generación de estereotipos y de estigmatizaciones del exogrupo ocurre en un escenario de supuesta competición social, regularmente aceptado mediante un discurso ideológico justificador.

Castañeda y Contreras (2012) sostienen que la discriminación social "no sólo son actitudes o conductas de desprecio, violencia o maltrato, sino que también comporta omisión y exclusión debida a la pertenencia a determinado grupo, el cual tiene asignado un estigma o desprestigio social" (p. 486). Se sostiene también que

la discriminación social que se ejerce hacia grupos en situación de vulnerabilidad es un fenómeno cada vez más extendido — aunque se tienda a negarlo — en la sociedad mexicana. Tal discriminación se expresa de distintas formas que van desde la negación y la indiferencia hacia ciertos grupos, hasta las prácticas de exclusión social y los crímenes por odio. (González, 2015b, p.10)

Otra definición de la discriminación, que nos servirá para enfocar nuestro estudio, es la siguiente:

es un fenómeno complejo que se manifiesta [...] en exclusión y falta de cohesión social, y [...] en representaciones socioculturales, estereotipos, tradiciones y estigmas, así como en manifestaciones simbólicas de inequidad que no necesariamente tienen un vínculo directo con las condiciones materiales. (Székely, 2006, p. 1)

Este fenómeno se fundamenta en la creación injusta de distinciones que forman parte de la identidad social de ciertos grupos integrados por "origen étnico o nacional, género, edad, discapacidad, religión, condiciones de salud, opiniones, condición social 
o económica, estado civil, preferencias sexuales, así como cualquier otra que atente contra la dignidad humana" (Soberanes Fernández, 2010, p. 263).

En un estudio sobre discriminación social llevado a cabo con adolescentes de bachillerato en varias regiones de México, se encontró que la discriminación social se expresa en diferentes formas:

Se hallaron al menos tres tipos diferentes de representaciones sociales de la discriminación: una que podríamos denominar conductual y que está integrada por comportamientos que denigran a las personas como: menospreciar, maltratar, separar, excluir, bromear, ejercer violencia, burlarse, entre otros [...] Otro tipo de representación social de la discriminación es el que podemos nombrar dirigido a grupos. En él los sujetos se orientan a reconocer la discriminación a los grupos que la sufren. Los conceptos que le son característicos son: pobres, negros, homosexuales, entre otros [...] Se identificó un tercer tipo de representación social de la discriminación, al que se le podría denominar por condición social, ¿en el que las comparten? consideran, más que las conductas y los grupos, las circunstancias sociales que generan la discriminación. Las nociones que las integran son: situación económica, ignorancia, color de la piel, entre otros. (González, Contreras \& Godínez, 2014, p. 170)

La discriminación social es, pues, una postura principalmente grupal, con fundamento en información escasa y el pensamiento automático (estereotipos, prejuicios, creencias, atribuciones) que son aplicados de manera irreflexiva para ofender, humillar y excluir a miembros de grupos diferentes con la intención de conservar una distancia social que favorezca al endogrupo, sin considerar los altos costos psicológicos y sociales de tales actos.

En lo que se refiere propiamente a la discriminación en la web, se podría establecer que la llegada del Internet a la vida cotidiana de las personas trajo consigo la certeza de que nuevas relaciones sociales podrían establecerse en una sociedad orientada a compartir información para crear conocimiento abierto. Después de 25 años observamos que, si bien el Internet ha traído muchos avances para la sociedad, también ha repetido y potenciado formas negativas de relación cara a cara de la vida cotidiana.

Uno de los problemas provocados por el avance del Internet ha sido el de la brecha digital entre diferentes grupos sociales, por lo que algunos autores (Abad, 2014, 2016; Abott, 2007; González, González \& Sánchez, 2013; Pellerin, 2013; Pérez \& Soto, 2014; Willemse, van der Velden \& Pot, 2015) mencionan la importancia de establecer una "e-inclusión" que emerge como un concepto importante ante la demanda de in- 
clusión y justicia sociales en la vida digital, que permita ampliar, y no segregar, la participación igualitaria, en la red, de diferentes grupos minoritarios que se diferencian por género, sexualidad, raza, edad, clase o discapacidad.

Trujano, Dorantes y Tovilla (2009) afirman que el Internet como espacio de interacción abierto ha significado una expansión de la violencia: "debido a la pluralidad de los contextos de participación del Internet, y a que modifica la manera de relacionarse los unos con los otros" (p. 9).

Los autores referidos sostienen que en Internet la violencia se manifiesta masivamente, desde el anonimato, de muchas maneras en la vida social en terrenos sensibles como la sexualidad, la desigualdad económica, la diversidad política y la estabilidad psicológica, lo que configura un nuevo escenario que se constituye en un reto para las disciplinas sociales.

Una aproximación interesante al problema de la discriminación, los mensajes de odio y el derecho que tienen las minorías a no ser agredidas en la web, la proporciona Kaufman, quien establece que el Internet no es un libro ni un blog, sino un sitio de interacción de personas de carne y hueso que se exponen a recibir agresiones que los orillan a salir del espacio virtual. El autor indica: "Internet [...] se transforma en parte de la misma sensación de vivir en sociedad, es decir, la tecnología nos ha dado la posibilidad de desarrollar nuestra experiencia humana conectándonos en permanencia con los otros sin límites temporales ni geográficos ni culturales" (Kaufman, 2015, p. 22).

Más adelante el autor menciona que:

el problema de Internet en realidad es más grave que la ausencia de regulaciones pertinentes a sus características: Internet es una práctica colectiva y global, resultante del desarrollo tecnológico, que constituye un territorio virgen de teorías éticas siquiera mínimamente aceptadas por sus habitantes sobre las cuales construir cualquier clase de regulación. La construcción de normas es harto dificultosa dado que, si bien la práctica es universal y se apoya sobre tecnologías idénticas y lenguajes informáticos comunes, las culturas y normas de sus participantes son tan diversas como los innumerables grupos sociales de nuestra humanidad digitalmente interconectada, pero además porque una mayoría de sus visitantes se encuentra a gusto en un espacio caracterizado por la anomia. (Kaufman, 2015, p. 31)

Abbott (2007) señala que "dentro de los círculos de investigación, la e-inclusión está vinculada con el estudio del movimiento de las discapacidades y el énfasis de encon- 
trarla en temas como la cultura, la autonomía y la identidad" (p. 6) que se refiere a la aplicación de la tecnología digital para reducir brechas digitales en ámbitos de género, desigualdad económica, generacionales y de sexualidad.

Cabero Almenara (2016) identifica varias acciones que deberían formar parte de esta inclusión tecnológica que remontaría la discriminación y la brecha digitales, y que son propias de la educación a distancia mediada tecnológicamente:

determinadas personas que posean algunos tipos de discapacidad; auxiliar con ella la escolarización de determinadas etnias y comunidades indígenas que se ven marginadas para acceder a la educación, favorecer una educación de calidad a zonas rurales con déficit de escuelas y de maestros, atender a poblaciones vulnerables y en situaciones de riesgo, llevar la formación a personas privadas de libertad, favorecer la integración de la formación de las mujeres, cobertura a poblaciones trabajadoras, proporcionar atención a personas que se hallen en el extranjero y facilitar la educación a personas en situaciones hospitalarias. (p. 3)

La normatividad del Internet sugerida por Kaufman (2015) es un gran dilema, ya que plantea la regulación de un medio abierto y originalmente democrático, para dar certeza y seguridad a sus participantes. Aunque las plataformas tecnológicas educativas no son totalmente abiertas, sí es verdad que en ellas se reproducen comportamientos discriminatorios y de exclusión que deben normarse para preservar el medio, el respeto a las minorías, y para que la plataforma cumpla con su función educativa igualitaria.

Rodríguez (2013) llama la atención sobre la necesidad de educar en Internet en tres aspectos: en el conocimiento de su diversidad y las posibilidades que representa; discutir y mostrar sus peligros y hacer una reflexión sobre su importancia y beneficios en la vida personal y cotidiana. Establece el autor: "sería necesaria una formación humanista y técnica sobre Internet. Este aprendizaje se dirigiría a profundizar en el conocimiento crítico de la red — posibilidades y amenazas - y a la formación de usuarios activos de Internet” (p. 165).

Entonces se puede sostener que la movilización de los usuarios de espacios en Internet para combatir, por medio de la inteligencia colectiva, a la exclusión, la etiquetación y toda forma de discriminación, será indispensable en el futuro, tal como señala Capelo (2015) "La campaña \#SinTags afirma que lo ideal sería fomentar una dignidad online, respeto a la diversidad, libertad del discurso, educación en derechos humanos, diálogo cultural e intercultural, educación en los medios electrónicos, activismo online [...] ejecutados por una ciudadanía democrática” (p. 151). 


\section{Método}

\section{Objetivo}

Identificar la posible existencia de discriminación social en las plataformas de interacción de la comunidad académica (profesores y alumnos) de la carrera de Psicología a distancia.

\section{Definición de discriminación social}

En el cuestionario de investigación se hizo explícito que la discriminación social debería entenderse como conductas o actitudes negativas grupales tendientes a la exclusión o al maltrato, provenientes de alumnos, profesores, personas externas o la administración escolar.

\section{Pregunta de investigación}

¿Existe discriminación social en las plataformas de interacción de la comunidad académica (profesores y alumnos) de la carrera de educación a distancia?

\section{Variables}

Se consideró como variable dependiente la percepción de discriminación social, y las variables independientes, al sexo, edad y sede de procedencia.

\section{Muestra}

La muestra estuvo integrada por estudiantes, 488 mujeres $(72.5 \%)$ y 185 hombres (27.5\%), mediante un muestreo incidental por convocatoria abierta. El 59\% representó a la sede metropolitana de la Facultad de Estudios Superiores de Iztacala, 12\% a Chimalhuacán, $6.7 \%$ a Oaxaca, $6.5 \%$ a Puebla, 6.1\% a Ecatepec, $4.2 \%$ a Tlaxcala, $4.2 \%$ a Toluca, 1.2\% a Tlaxcoapan yo.1\% a San Felipe Orizatlán. La edad promedio fue de 36.7 años.

Es importante establecer que el estudio, dado sus límites de diseño y propósitos, no pretendió alcanzar representatividad y significatividad estadística alguna, sino 
reconocer la emergencia de discriminación y exclusión, para poder identificarlos y atenderlos.

\section{Instrumento}

Se diseñó y aplicó un formato de Google en línea para capturar las respuestas a 20 ítems sobre discriminación considerando los siguientes campos de información: discriminación en espacios de aprendizaje, roles en la discriminación, testigos de relaciones discriminatorias y razones de esas conductas.

\section{Resultados}

\subsection{Resultados en el estudio cuantitativo sobre discriminación}

Para identificar qué tanto habían sido testigos los participantes en el estudio de la discriminación en espacios de aprendizaje mediados tecnológicamente, se incluyeron la plataforma educativa Moodle (utilizada en el suAyed), Facebook, páginas de Internet, foros y blogs, correo electrónico y Skype.

Se eligieron esos espacios de interacción para comparar la percepción de discriminación entre la plataforma institucional y los sitios de acceso abierto más comunes en Internet.

En la Figura 1 se observa que, en general, los estudiantes refieren no haber sido testigos de tratos discriminatorios. De cualquier forma, llama la atención que la plataforma Moodle sea la que tenga el porcentaje más alto, ya que $24.4 \%$ de los participantes mencionó haber visto discriminación en ella. Le sigue Facebook con un poco más de $20 \%$ y los espacios de interacción en Internet con $18.3 \%$.

Estos datos son relevantes ya que permiten tomar consciencia de que es en el espacio educativo institucional a distancia (en el que están establecidas normas de trato entre los actores educativos) donde ocurren más interacciones discriminatorias.

Posteriormente se indagó sobre las relaciones de discriminación en el ámbito escolar en la web. Se evaluaron las interacciones alumno-alumno, profesor-alumno y profesor-profesor. 


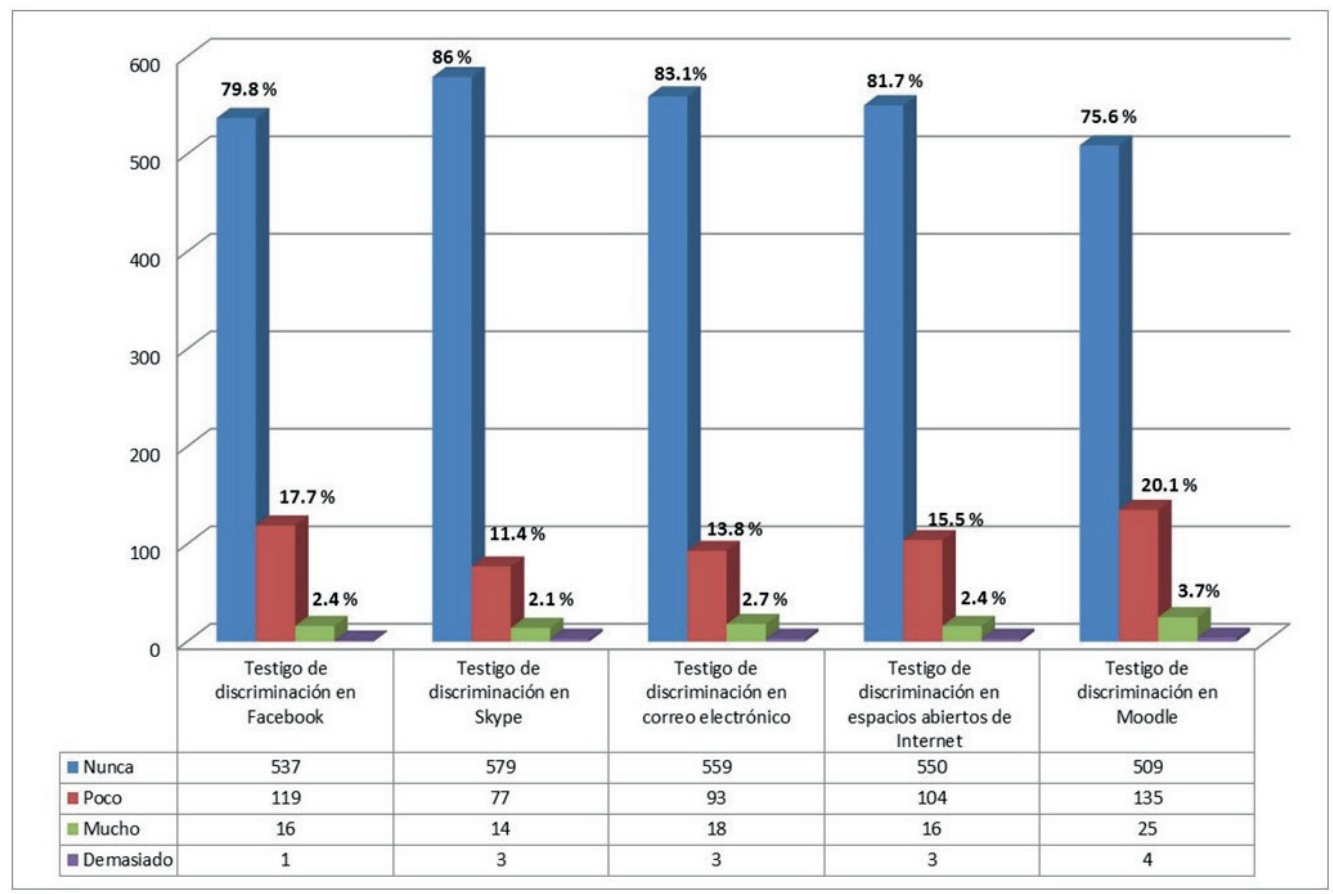

Figura 1. Discriminación en espacios de interacción en la web

Fuente: Elaboración propia.

Los resultados de la Figura 2 muestran que los entrevistados consideran que son las relaciones alumno-alumno y profesor-alumno, las que más discriminación expresan. Asílo indicó $22.7 \%$ de la muestra, en el primer caso, y 17.1\%, en el segundo. Aunque las cifras son relativamente bajas, sí es importante reconocer que la discriminación se expresa principalmente entre los pares de alumnos y desde el profesor al estudiante, con el objetivo de promover un trato igualitario y respetuoso entre estos grupos de la comunidad universitaria. 


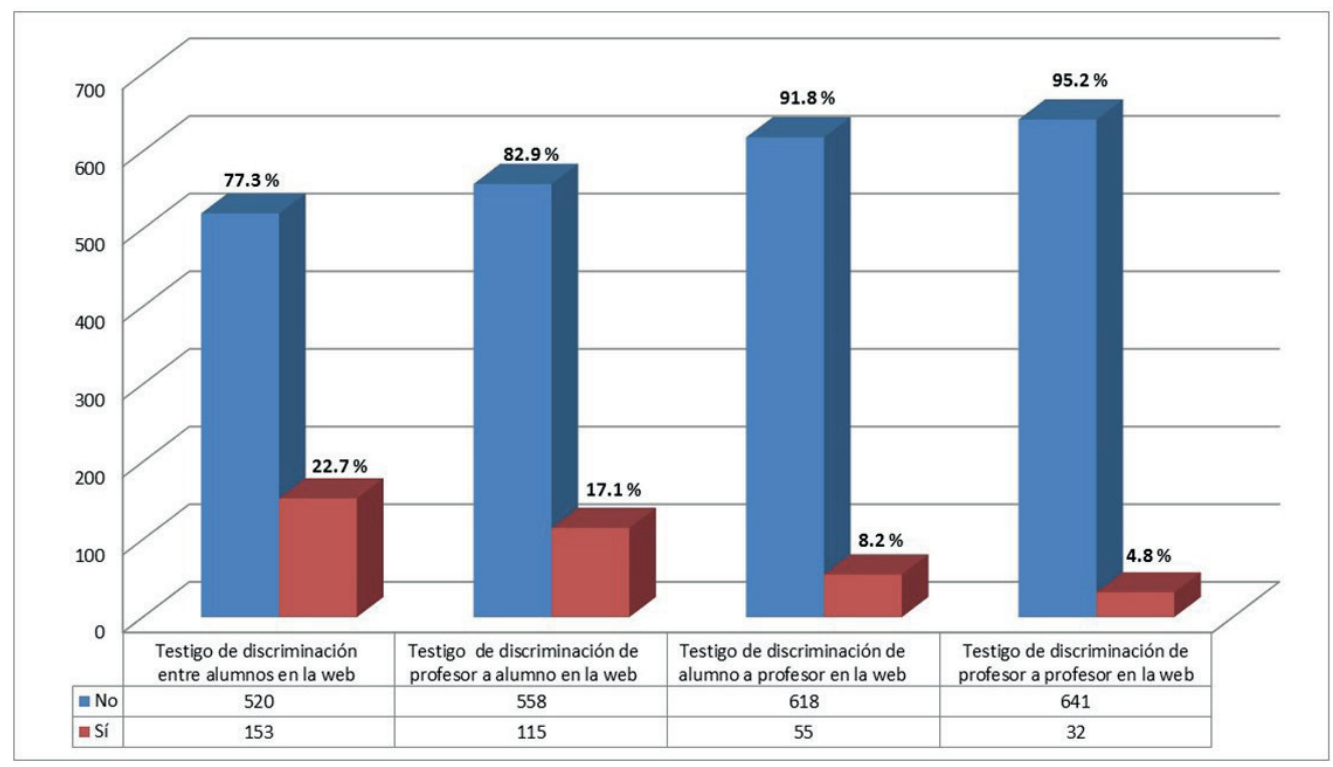

Figura 2. Testigos de discriminación en la web

Fuente: Elaboración propia.

Posteriormente se preguntó si alguna vez habían discriminado o se habían sentido discriminados en los espacios de interacción en línea. La Figura 3 muestra los resultados. En consonancia con otros estudios sobre discriminación, 96.1\% de nuestra muestra aseguró nunca haber discriminado a alguna persona utilizando los medios de interacción en línea, mientras que un porcentaje cercano al 20\% manifestó haberse sentido discriminado alguna vez.

Aunque la cifra sea menor, no deja de ser indicativa de la existencia del fenómeno de la discriminación social hacia los alumnos de la carrera de Psicología a distancia, por lo que es importante establecer medidas para reducirla y evitarla.

Finalmente, se interrogó a los estudiantes por las razones de haber sido discriminados y se encontraron porcentajes bajos en cada una de las causas mencionadas. La que puntuó más alto fue la forma de pensar, que manifestó haber sentido $15.3 \%$, seguido por la forma de expresarse, con $11.4 \%$ y por motivos de la edad con $10.4 \%$. 


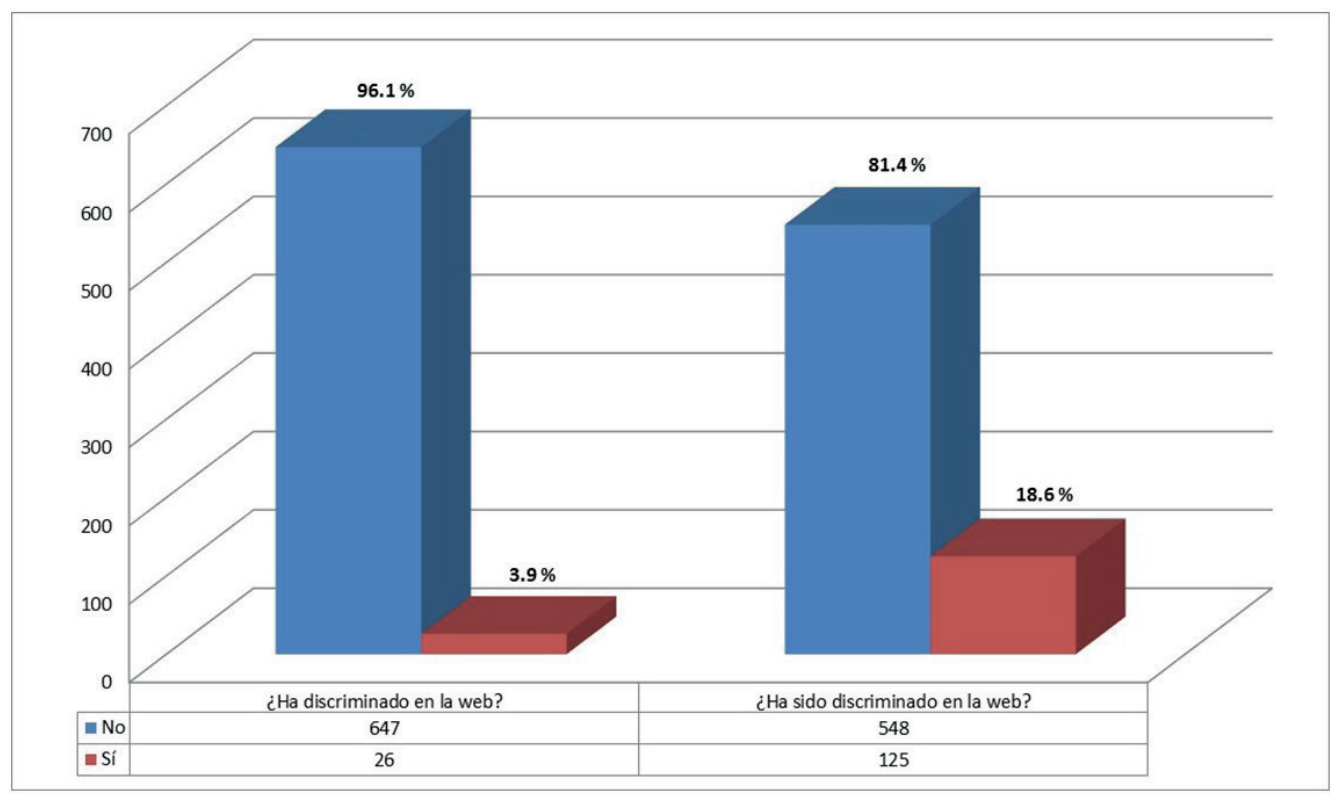

Figura 3. Discriminador/discriminado en la web

Fuente: Elaboración propia.

Es necesario señalar que se aplicaron pruebas ANOva para todas las combinaciones posibles de variables y a un nivel de significancia de $0.05 \%$; en la prueba confirmatoria Schefeé, los grupos de edad de 26 a 35 años y de más de 66 años mostraron diferencias significativas. Ampliando el nivel de significancia a $0.10 \%$, las diferencias estadísticamente significativas se encontraron entre los grupos de edad de 26 a 35 con los de 46 a 55 y más de 66 años; y de más de 66 con los grupos de 26 a 35, 36 a 45 y 56 a 65 años. Los grupos de más edad sienten mayor discriminación que los grupos de edades más jóvenes. Lo anterior nos indica que la edad es una característica sensible de discriminación (Figura 4). 


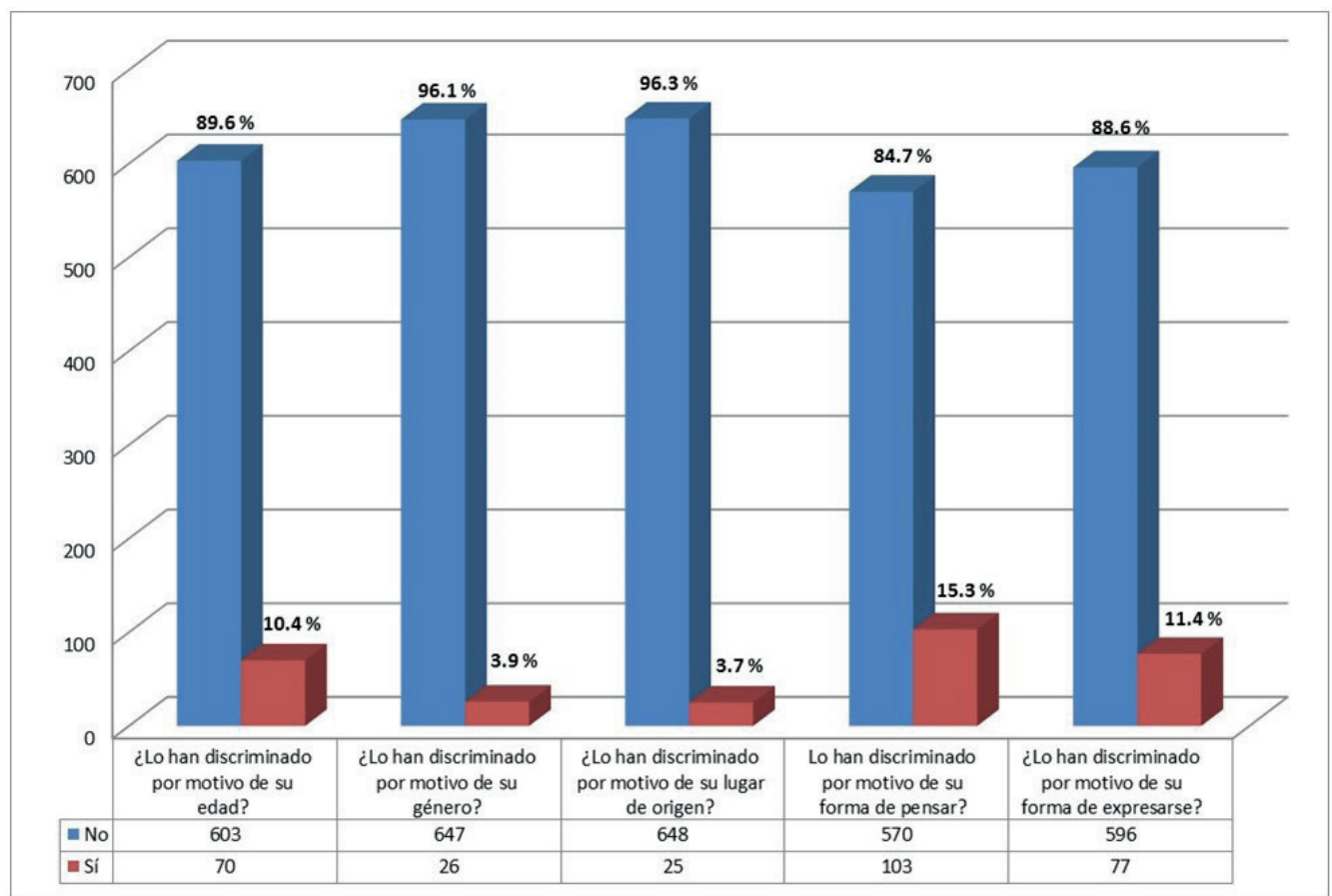

Figura 4. Motivos de discriminación en la web

Fuente: Elaboración propia.

\subsection{Resultados en el estudio cualitativo sobre discriminación}

Para complementar el estudio cuantitativo basado en los reactivos del instrumento diagnóstico sobre discriminación, se pidió a los integrantes de la muestra referir algún caso de discriminación vivido en el SUAyed. Las participaciones abiertas proporcionaron una mejor comprensión del problema. Se obtuvieron respuestas de 156 sujetos.

Es importante mencionar que se identificaron varios tipos de discriminación del profesor hacia el alumno (37\%), discriminación del alumno hacia otro alumno (36\%) y discriminación externa hacia el suayed (14\%). En la Figura 5 se observan todas las categorías de discriminación, sus frecuencias y porcentajes. Aparecen entre comillas las referencias textuales obtenidas de los participantes. 


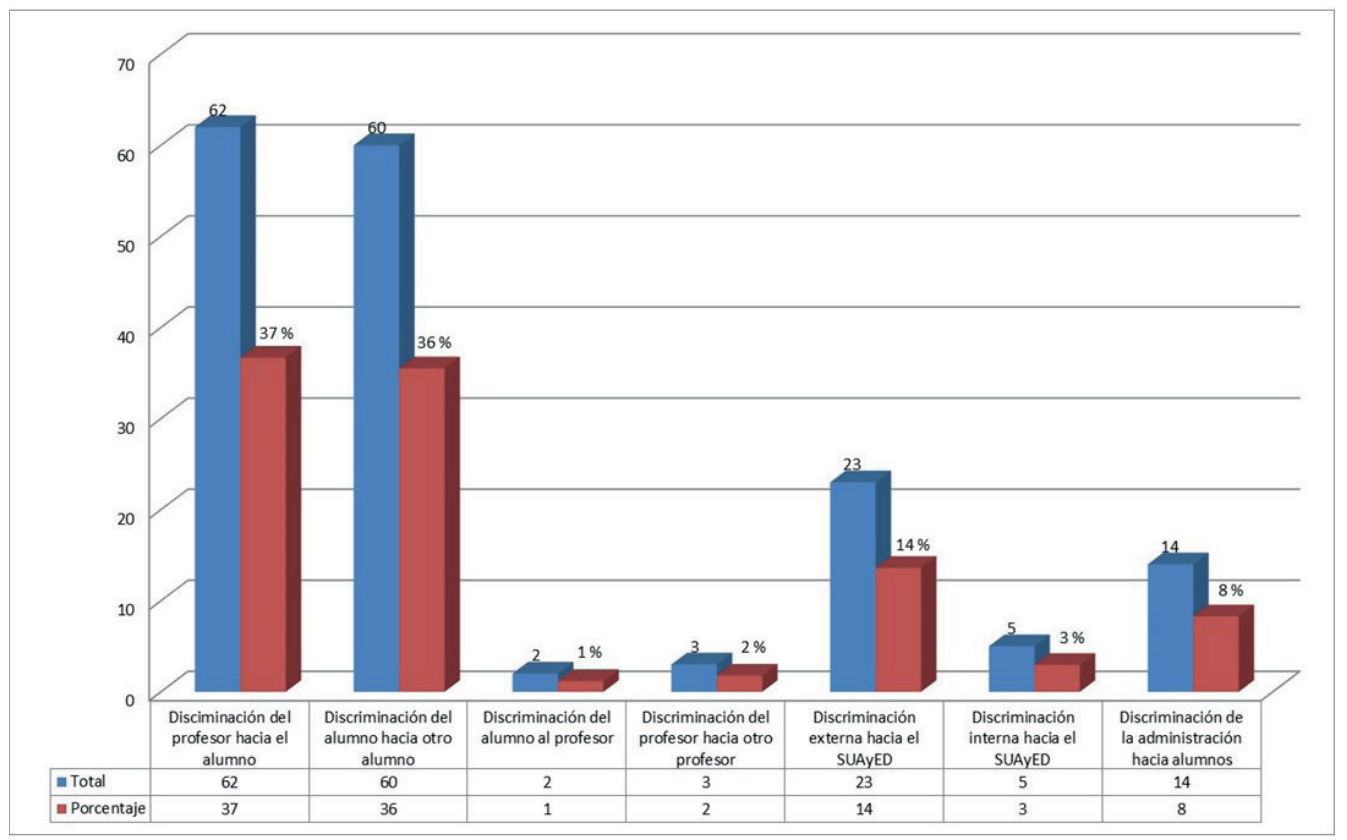

Figura 5. Casos de discriminación en el SUAyED

Fuente: Elaboración propia.

Dentro del rubro discriminación del profesor hacia el alumno, los participantes en el estudio refieren lo siguiente: ignorar al estudiante cuando plantea dudas o solicita correcciones; no dar seguimiento a las actividades; no contestar correos electrónicos; tomar represalias por comentarios; tener un trato agresivo contra los estudiantes; usar lenguaje sexista y mostrar favoritismo hacia algunos alumnos.

Ejemplos de este tipo de discriminación son los comentados en los siguientes testimonios:

Le hice a un profesor preguntas respecto a la forma de trabajo. Se molestó y al final me puso en el módulo NP en cuarto semestre, lo cual repercutió que volviera a recursar y perder un semestre por una materia. ¡No se les puede decir nada!, aunque ellos no califiquen ni retroalimenten nuestros comentarios. (Sujeto 121)

Dejé de estudiar por muchos años y ahora regreso a la universidad, obviamente estoy en desventaja en el uso de las TIC y envié mensaje a Skype a mi asesora para pedirle un consejo sobre el uso de una herramienta y fui ignorada. La tengo como 
asesora y la necesité apenas la semana pasada para una información sobre una tarea y me volvió a ignorar, cuando yo sé que ella estaba activa en Skype. Última vez que la contacto. (Sujeto 31)

En la Figura 6 se muestra la discriminación del profesor hacia el alumno.

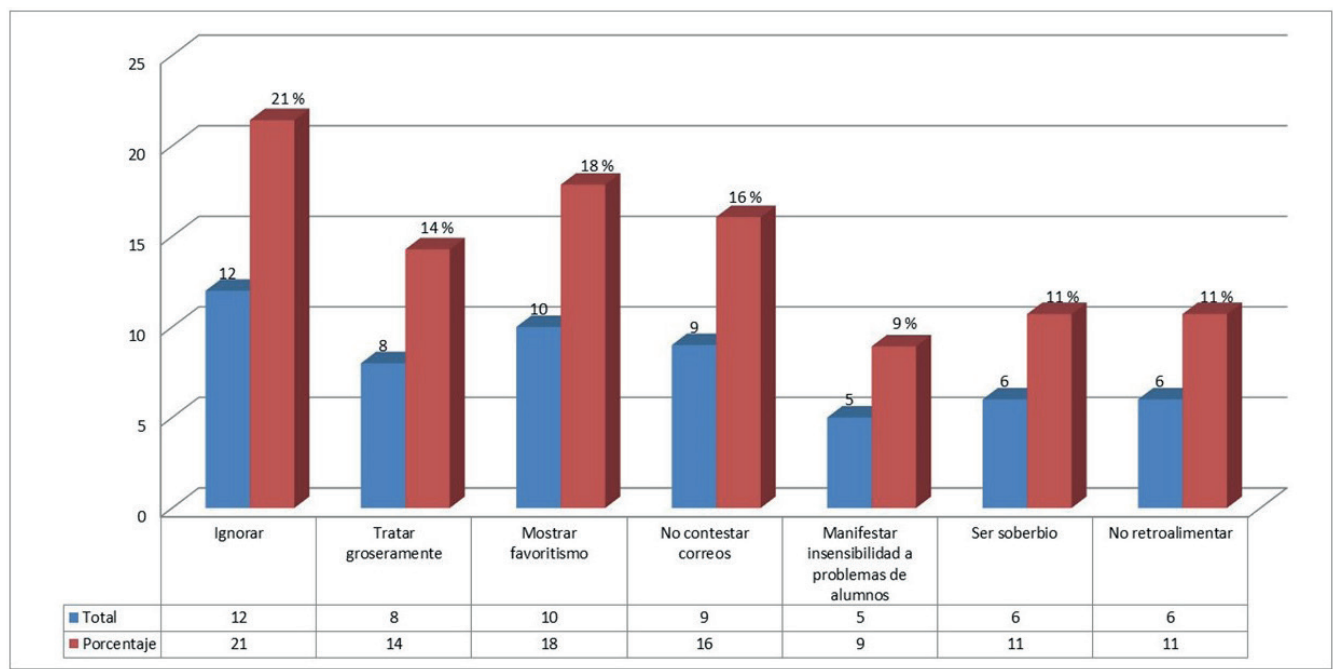

Figura 6. Discriminación del profesor hacia el alumno

Fuente: Elaboración propia.

De los participantes que expresaron sus experiencias en esta categoría, $21 \%$ identificó que el ser ignorado es el principal comportamiento del profesor que lo hace sentirse discriminado. Posteriormente, con $18 \%$, que el docente muestre favoritismo por otros estudiantes; con $16 \%$ que no conteste correos y $14 \%$ tratar groseramente a sus alumnos.

En lo que se refiere a la categoría discriminación del alumno hacia otro alumno (Figura 7). Se identificaron los siguientes comportamientos como los más frecuentes: se integran equipos de trabajo sólo con amigos o estudiantes de la misma sede (27\%); se hacen diferencias por el conocimiento previo que se tiene en la materia (23\%) y exclusión por razones de edad (20\%). 


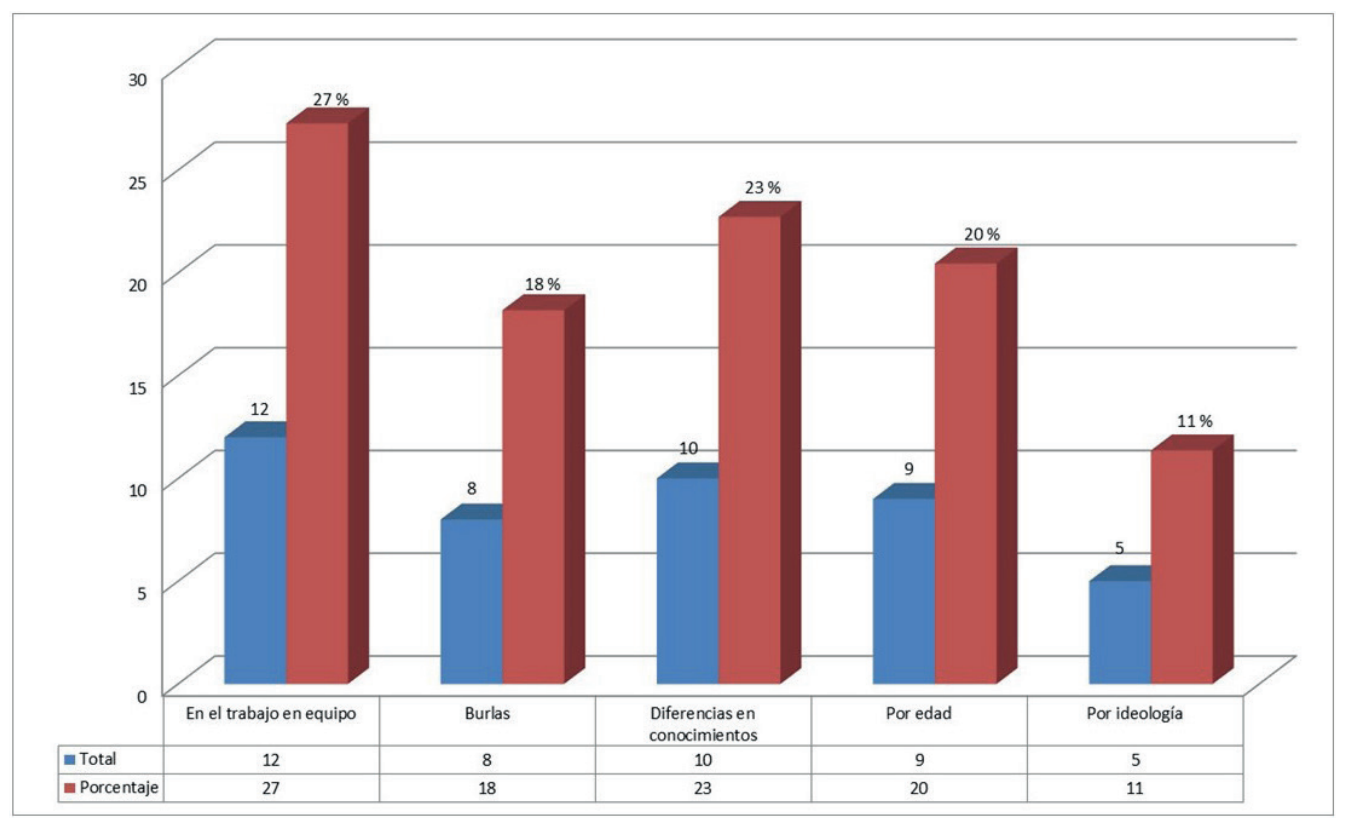

Figura 7. Discriminación del alumno hacia otro alumno

Fuente: Elaboración propia

Algunos testimonios de los participantes nos muestran claramente estas situaciones:

En trabajos en equipo, por mi edad creen que soy inútil o que sólo puedo hacer cosas sencillas y se adelantan a realizarlas ellos. Me ha pasado que no toman en cuenta mi opinión. Me ignoran. En ocasiones me preguntan: ¿Por qué estás estudiando a tu edad?, en tono de burla. O cuando voy a la Fes Iztacala, la gente se adelanta a preguntarme si soy mamá de algún estudiante. Cuando dices que eres estudiante, no lo creen. (Sujeto 84)

En el momento de hacer los equipos de trabajo, se cierran los pequeños grupos, porque son conocidas entre sí, por la edad u otras afinidades. Y en la construcción de wikis, predominó la redacción de una compañera, quien llegó a escribirme a mi correo personal y me pidió que dejara de participar porque modificaba la redacción y no se entendía, y de paso, se desconfiguraba el formato, que a ella le había costado mucho conservar. (Sujeto 17) 
Es importante hacer notar que $20 \%$ expresó que la edad es un factor que en las relaciones cotidianas de los estudiantes predispone a la discriminación. Estos datos urgen a los profesores a establecer procedimientos de trabajo en equipo que cancelen la exclusión e integren a los estudiantes independientemente de su edad o formación académica previa.

En cuanto a la discriminación de alumno hacia un profesor, la discriminación de profesor hacia otro profesor y la discriminación interna en el SUAyED se observa que son poco frecuentes, lo que indica que su existencia podría considerarse marginal.

Las distintas formas en las que se discrimina externamente al SUAYED, obtenidas de los testimonios, se muestran en la Figura 8.

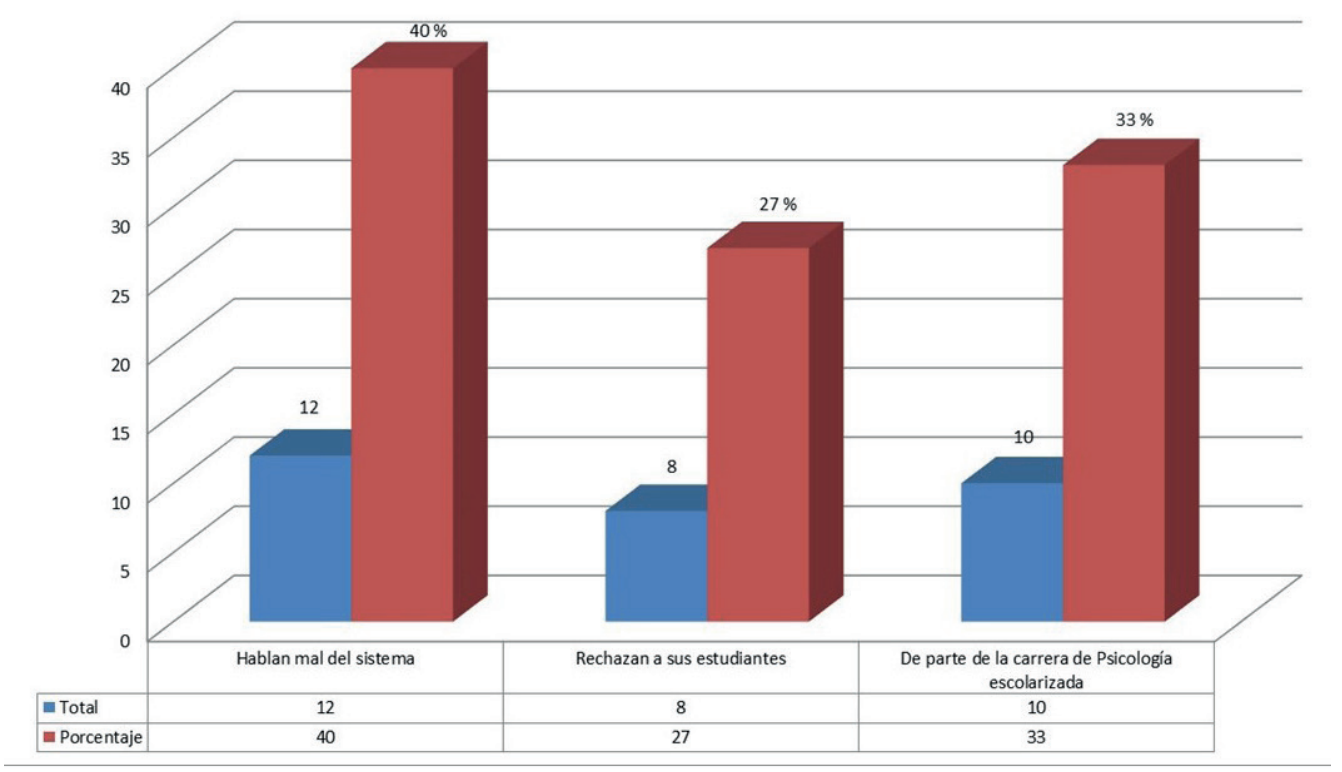

Figura 8. Discriminación externa hacia el SUAyED

Fuente: Elaboración propia.

Se identificaron opiniones negativas o conductas de exclusión provenientes de miembros de instituciones externas, entre ellas: opiniones estereotipadas de que la educación a distancia es de baja exigencia académica y nula calidad (40\%); exclusiones provenientes de miembros de la carrera de Psicología escolarizada de la FEs Iztacala 
(33\%) y rechazo de algunas instituciones hacia alumnos que llevan a cabo sus prácticas, por pertenecer a un sistema educativo a distancia, y etiquetaciones basadas en el escaso conocimiento que se tiene del suAyED en la sociedad (27\%).

Algunos de los testimonios expresados por los estudiantes en esta categoría son los siguientes:

En un ciclo de conferencias, un alumno de Psicología del sistema escolarizado comentó que no podía compararse el nivel de preparación que ellos tenían, el cual consideraba mejor, al de los alu|mnos de SUAyED, porque consideraban que la preparación es de más calidad. En esa ocasión, un docente del escolarizado comentó que él no creía en los alumnos de suAyed y que, cuando les daba clase, no les daba el máximo de calificación en las tareas, porque decía que no podía constatar la forma en que las hacían y dudaba que egresaran lo suficientemente preparados, como los alumnos del escolarizado. (Sujeto 77)

En una práctica el director de la escuela me preguntó si yo era fósil de la UNAM debido a mi edad; por cierto, nunca me dio respuesta de si podía realizar la práctica en esa institución a pesar de cumplir con todos los lineamientos que requería la práctica. (Sujeto 68)

El problema de discriminación identificado muestra que es importante trabajar en el prestigio social del SUAyED y tomar acciones para hacerlo más visible. Resulta sorprendente que sean algunos miembros de la carrera de Psicología presencial quienes compartan estos argumentos discriminatorios, a pesar de estar en la misma universidad.

Finalmente, se identificó otra categoría a la que se denominó discriminación de la administración hacia los alumnos, y que incluye a los diferentes niveles de administración escolar de la UNAM. La información compartida en la Figura 9 señala que la principal actividad discriminadora ejercida por la administración, con $36 \%$ de menciones, es la de realizar actividades académicas que sólo benefician a los estudiantes de la ciudad y, dado que esos eventos no se transmiten en línea, resulta excluyente para los alumnos de provincia. El 21\% indica que el principal problema es la falta de respuesta oportuna de parte de la administración a las quejas interpuestas por los estudiantes por incumplimiento en las tareas de los profesores, y $14 \%$ considera que no hay respuesta expedita cuando se solicita apoyo tecnológico en problemas de conexión o plataforma. 


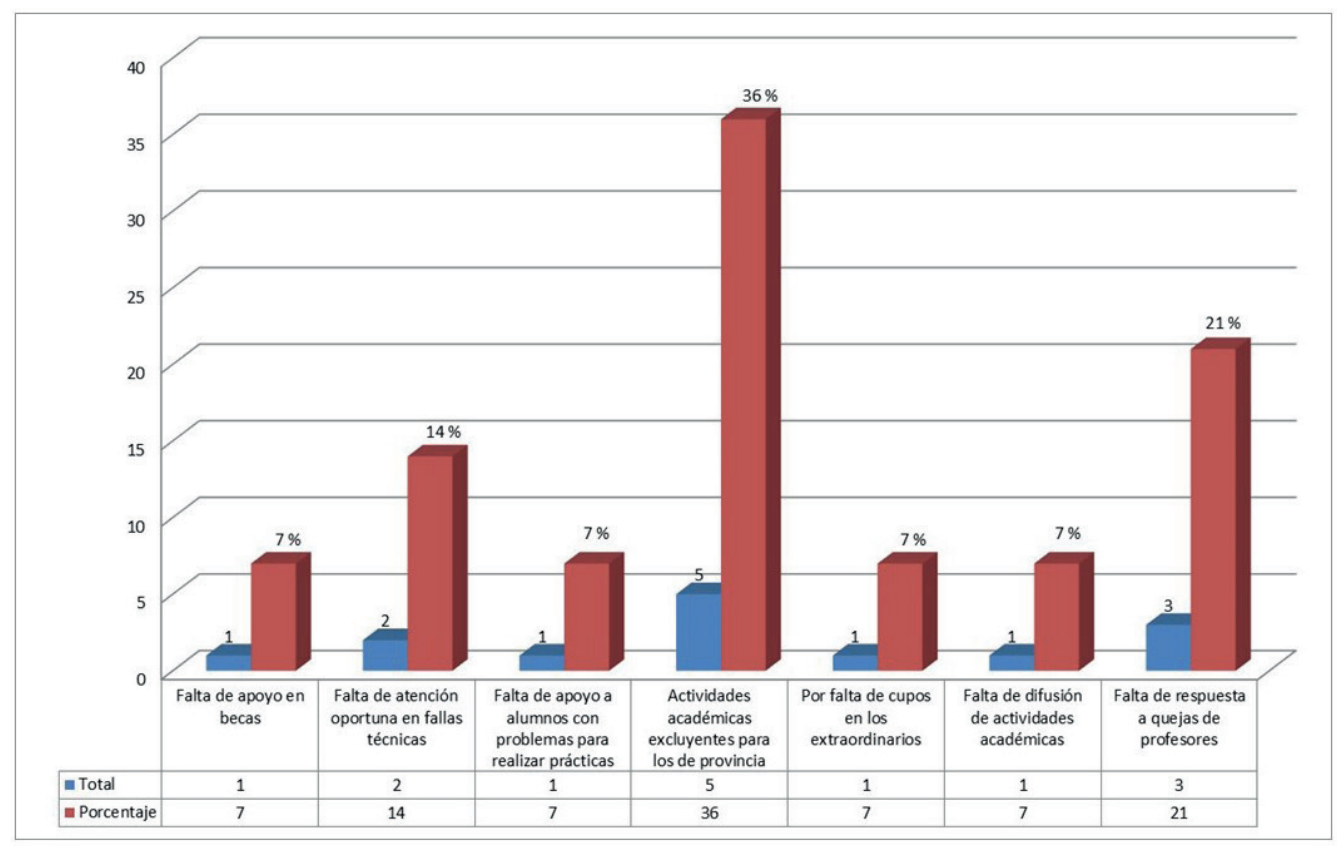

Figura 9. Discriminación de la administración hacia los alumnos

Fuente: Elaboración propia.

Estos datos evidencian que hay un sentimiento de discriminación en los estudiantes porque no son considerados en sus necesidades y peticiones para poder avanzar y mejorar en su formación profesional. La administración escolar debe reconocer esta situación para implantar una estrategia de mejora en el otorgamiento de servicios escolares.

\section{Análisis de los resultados}

La realización de este estudio diagnóstico brindó la oportunidad de conocer cómo se generan y difunden comportamientos discriminatorios y excluyentes entre los miembros que conforman el suAyed Psicología Iztacala; es decir, alumnos, profesores, administrativos y agentes externos. La investigación empírica interrogando a los estudiantes (participó alrededor de $20 \%$ de la matrícula total) mostró que el proble- 
ma de discriminación no está generalizado ni es alarmante, pero tiene presencia en el sistema, ya que $20 \%$ de los participantes afirmó haberse sentido discriminado alguna vez en el SuAyed, y que es en las aulas virtuales, en la plataforma Moodle, donde más ocurren estas conductas, por encima de Facebook y otros espacios en Internet.

Los estudiantes también señalaron que las interacciones en las que existen relaciones de discriminación de una manera más evidente son de alumno a alumno y de profesor a alumno (la parte cuantitativa del estudio identificó a la primera como la más predominante en discriminación, y la parte cualitativa los colocó prácticamente en igualdad de incidencia). Las relaciones de discriminación de alumno a alumno se presentan, principalmente, en la organización y desarrollo de las actividades en equipo, ya que se muestra amiguismo; favoritismo hacia las opiniones de los que más saben; discriminación por orientación sexual, lugar de procedencia y edad, y exclusión de la integración del trabajo por considerar de poca valía las contribuciones de otros compañeros. También expresaron, tal como se encontró en el ANOvA aplicado en la parte cuantitativa, que la edad es un factor de discriminación entre los estudiantes y también señalaron las diferencias de conocimiento como un elemento discriminador, ya que se valoran más las participaciones de compañeros que tienen otras licenciaturas o posgrados.

En cuanto a la discriminación de profesores a alumnos, los testimonios de los estudiantes indicaron que la principal manifestación es el ser ignorados cuando se les pide retroalimentación o consejos en Moodle u otras vías, y cuando se hacen aportaciones a la clase o propuestas que no son tomadas en cuenta. Otro caso de discriminación se da cuando el profesor muestra favoritismo hacia algunos alumnos, en detrimento de otros, por razones de edad, sexo, formación previa o por agrado personal.

Otra forma de sentirse discriminado es la que proviene del medio externo. Los participantes del estudio indicaron que la principal forma de expresión discriminadora es cuando personas ajenas al suAyed hablan mal de él al aplicar un estereotipo negativo de la educación a distancia. Otra manifestación proviene del rechazo de personas que representan instituciones para que los estudiantes de Psicología a distancia puedan realizar sus prácticas en esos lugares, por desconocimiento del sistema o por la desconfianza que genera la edad madura de los posibles practicantes.

También resulta revelador saber que, a juicio de los estudiantes, la administración realiza actos de discriminación cuando excluye a los alumnos que presencialmente no pueden estar en las diversas actividades académicas llevadas a cabo en la FEs Iztacala, y cuando no atiende a los estudiantes que se quejan del profesor por incumplimiento de sus actividades y por demandas de asesoría técnica. 


\section{Conclusión}

La realización de la investigación y, particularmente, el análisis cuantitativo y cualitativo de los resultados, nos permite identificar la existencia del fenómeno de la discriminación en las plataformas tecnológicas de interacción de la comunidad del suAyED y sus diversas particularidades.

Es evidente que para atajar el incremento y promover la disminución y eventual supresión de las diferentes formas de discriminación, la administración de la carrera debería llevar a cabo una serie de acciones, como las siguientes, derivadas de los fenómenos observados en el estudio:

1. Establecer una campaña de información que posicione al suayed Psicología como una dependencia libre de cualquier forma de discriminación social y, al mismo tiempo, adoptar como suyos los valores de la tolerancia, el respeto y la inclusión.

2. Generar una normatividad que regule las formas de trato en la plataforma tecnológica Moodle.

3. Capacitar a los profesores en el uso de técnicas didácticas colaborativas que permitan la participación de todos los miembros de los equipos en el desarrollo de sus actividades académicas. Es importante que los profesores den el seguimiento oportuno y la asesoría necesaria al trabajo colectivo de los alumnos.

4. Dentro de la campaña de información señalada anteriormente, es menester hacer referencia a la tolerancia a la variabilidad de edades de los alumnos que estudian Psicología a distancia, promover la apertura para su inclusión y aprender unos de otros.

5. De la misma manera señalar, que, independientemente de la formación académica anterior de los estudiantes, todos están en el mismo proceso de aprendizaje de la psicología, en igualdad de circunstancias.

6. Es importante hacer hincapié en los profesores que el impacto que se genera al ignorar las peticiones de los estudiantes o no contestar sus correos, es altamente desmotivador, en especial en la educación a distancia, donde la interactividad permanente debe ser una característica. Dentro de los lineamientos de trabajo del profesor se debe incluir la obligatoriedad de la respuesta oportuna.

7. El favoritismo es una cara opuesta a la discriminación. Se hace necesario hacer conciencia entre alumnos y profesores que esa práctica debe ser anulada para cuidar la igualdad y equidad entre los estudiantes.

8. La discriminación externa hacia al SUAyED debe ser contrarrestada por las más altas dependencias de la UnAM. En lo que compete a la administración del suAyed, 
requiere hacer una campaña de visibilidad sobre los alcances del sistema educativo a distancia, para ganar prestigio social. También es indispensable que ante la exclusión de alguna dependencia hacia los alumnos que desean hacer prácticas supervisadas, se establezca contacto con la institución para asegurarle la calidad del servicio académico proveniente del alumno. Firmar convenios de colaboración entre instancias iguales podría funcionar para evitar discriminación y exclusión.

9. Tener intercambios y participar en eventos académicos conjuntos con la carrera de Psicología escolarizada de la FEs Iztacala impactará en la reducción de los estereotipos que algunos de sus miembros comparten sobre la calidad de estudios impartidos en el SUAyED.

10. La administración, representada por la Coordinación de Educación a Distancia de la fes Iztacala, requiere llevar a cabo una gestión psicosocial del suAyed Psicología, ya que se deben reconocer las diferentes condiciones de los alumnos que se encuentran en distintas zonas del país y en el extranjero y tratar de reducir esas brechas, estableciendo condiciones de igualdad y equidad. Transmitir los eventos académicos en línea ya sea sincrónica o asincrónicamente, es una manera de salvaguardar esa igualdad. Por otra parte, dar respuesta oportuna a las solicitudes, comentarios y quejas a través de la implantación de una mesa de ayuda en la plataforma, es una necesidad en la gestión de asuntos que todo sistema de educación a distancia debe tener.

\section{Alcances y limitaciones}

Para finalizar, sólo resta reflexionar sobre los alcances de esta investigación y las posibilidades que a futuro se presentan, para generar un mayor conocimiento sobre la discriminación y la exclusión que ocurren o podrían ocurrir en la interacción de los diferentes espacios virtuales en la educación superior a distancia en México.

Los resultados muestran que algunas formas antisociales que se expresan en aulas y espacios en la educación presencial, se repiten en las interacciones mediadas tecnológicamente, en aulas virtuales que suponen horizontalidad en las relaciones entre los participantes del proceso educativo.

Este estudio podría ampliarse para investigar las relaciones entre alumnos a distancia y mejorar sus habilidades sociales como la empatía, la tolerancia y el respeto hacia los otros. Estudiar las relaciones entre profesores y estudiantado para perfeccionar las competencias docentes que impacten en la formación educativa del estudiante a 
distancia y, finalmente, dotar a las administraciones escolares de conocimiento sobre cómo se presentan relaciones de discriminación y exclusión en sus aulas de aprendizaje, para establecer acciones que promuevan la tolerancia y el respeto.

\section{Lista de referencias}

Abad, L. (2014). Media literacy for older people facing the digital divide: The e-Inclusion programmes design. Comunicar. Revista Científica de Comunicación y Educación, 21(42), 173-180. doi:http://dx.doi.org/10.3916/C42-2014-17

Abad, L. (2016). La alfabetización digital como instrumento de e-inclusión de las personas mayores. Prisma Social, 16(2), 156-204.

Abbott, C. (2007). E-inclusion: Learning difficulties and digital technologies. FutureLab (Report15), 1-36.

Cabero, J. (2016). La educación a distancia como estrategia de inclusión social y educativa. Revista Mexicana de Bachillerato a Distancia, 15, 2-6. Recuperado de http://bdistancia.ecoesad.org.mx/?abstract=la-educacion-a-distancia-comoestrategia-de-inclusion-social-y-educativa

Capelo, M. C. (2015). Inteligencia colectiva para combatir manifestaciones de odio en Internet. En M. Campos, M. Ramos, R. Trejo, M. E. Hernández, S. Corona, C.Ávila... J. Caballero (Coords.), Mensajes de odio y discriminación en las redes sociales (pp. 146-160). México: Consejo Nacional para Prevenir la Discriminación.

Castañeda, L. I., \& Contreras, K. A. (2012). Jóvenes y discriminación: la naturalización de las violencias. Revista Electrónica de Psicología Iztacala, 15(2), 485503. Recuperado de http://www.revistas.unam.mx/index.php/repi/article/ view $/ 32364$

González, M. A. (2015a). Encuentros diagnósticos de tutores y alumnos en el suAyed Psicología de la fes Iztacala. En R. Freixas \& F. Ramas (Coords.), Buenas prácticas de educación abierta y a distancia (pp. 58-74). México: Coordinación de Universidad Abierta y Educación a Distancia-Universidad Nacional Autónoma de México.

González, M. A. (2015b). Sobre la discriminación social en México en población adolescente. Políticas públicas y análisis psicosocial. México: Fanguye/Coordinación de Educación a Distancia-Universidad Nacional Autónoma de México. 
González, M. A., Contreras, K., \& Godínez, N. (2014). Análisis de la representación social de la discriminación con base en la prueba de asociación libre de palabras con jerarquización. En M. A. González (Coord.), La discriminación social en México. Un estudio comparativo con base en la clase social, el sexo y la región del país (pp. 134-174). México: ImagiaComunicación/Instituto Tecnológico y de Estudios Superiores de Monterrey.

González, M. P., González, C. F., \& Sánchez, J. M. (Junio, 2013). Evaluation model of e-inclusion for Colombian context: border zone case. International Conference on Information Society (i-Society) de IEEE, Advancing Technology for Humanity, Toronto. Recuperado de https://www.spectronics.com.au/conference $/ 2010 / \mathrm{pdfs} /$ E-inclusion\%20-\%20Learning\%20Difficulties\%20and\%20 Digital\%20Technologies.pdf

Kaufman, G. A. (2015). Libertad de expresión y protección de grupos discriminados en Internet. México: Consejo Nacional para Prevenir la Discriminación.

Pellerin, M. (2013). E-inclusion in early french immersion classrooms: using technologies to support inclusive practices that meet the needs of all learners. Canadian Journal of Education, 36(1), 44-70.

Pérez, D., \& Soto, P. (2014). A virtual campus for E-learning inclusion; the case of svC-G9. Journal of Universal ComputerScience, 20(2), 240-253.

Rodríguez, R. (2013). Educar en Internet. Una propuesta para la construcción democrática de la red. Revista Internacional de Pensamiento Político, 8, 161-176.

Soberanes, J. L. (2010). Igualdad, discriminación y tolerancia en México. Cuestiones Constitucionales. Revista Mexicana de Derecho Constitucional, $22(1), 261-273$.

Székely, M. (2006). Un nuevo rostro en el espejo: percepciones sobre la discriminación y la cohesión social en México. Santiago de Chile: Comisión Económica para América Latina y el Caribe.

Tajfel, H. (1984). Grupos humanos y categorías sociales. Barcelona: Herder.

Trujano, P., Dorantes, J., \& Tovilla, V. (2009). Violencia en Internet: nuevas víctimas. Liberabit. Revista de Psicología, 15(1), 7-19.

Willemse, B., van der Velden, C., \& Pot, A. M. (2015). Barriers and needs in ICT use of older people. A transnational iAge study. Amsterdam: Neatherlands Institute of Mental Health and Addiction. 